\title{
Pillars of remyelination
}

In individuals with multiple sclerosis, the demyelination of axons leads to defective transmission of signals along nerve fibres. The development of drugs that can stimulate remyelination has been hampered by the lack of appropriate systems to screen compounds for such an effect in a high-throughput setting. Reporting in Nature Medicine, Mei and colleagues have now developed such a system and used it to identify a group of antimuscarinic compounds that enhance remyelination.

Multiple sclerosis generally has two phases. Most of the currently available drugs target the autoimmune processes that characterize the first, relapsing-remitting phase. During the subsequent progressive phase, the immune system has less of a role, the extent of recovery between relapses decreases and neurons become progressively demyelinated. Drugs that can promote remyelination would, therefore, help to address the substantial unmet medical need of patients with progressive multiple sclerosis.

Oligodendrocyte precursor cells (OPCs) are recruited to the site of demyelination, where they differentiate into oligodendroglia that remyelinate the axons. To investigate this process in cell culture, Mei et al. engineered conical micropillars of compressed silica to mimic unmyelinated axons. These micropillars were then incubated with OPCs, which differentiate into oligodendrocytes in culture. Multiple OPCs (which were identified by

staining for platelet-derived growth factor receptor- $\alpha$ ) associated with

both the number of micropillars ensheathed and the area covered by myelin could be observed from a single, twodimensional image

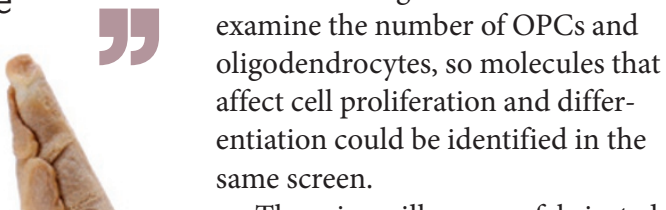

The micropillars were fabricated in a 96-well format, and 1,000 bioactive compounds were screened to identify molecules that promote myelination and oligodendrocyte differentiation. Most of the compounds tested reduced the number of cells of both types that associated with micropillars, which could be attributable to either the toxicity of the compounds or to reduced adhesion of the cells to the micropillars. Eight US Food and Drug Administration (FDA)-approved antimuscarinic compounds, including clemastine - a widely available first-generation antihistamine - increased the number of myelin-ensheathed micropillars and decreased the number of OPC-associated micropillars, suggesting that these compounds promote both oligodendrocyte differentiation and myelination.

The investigators then examined the effects of clemastine in a mouse model of multiple sclerosis, in which lysolecithin is used to induce neuronal injury. Oral administration of clemastine before the injury accelerated remyelination, increased the thickness of the regenerated myelin sheath and increased the percentage of remyelinated neurons 14 days after injury.

On the back of these experiments, a Phase II trial of clemastine for the treatment of patients with relapsingremitting multiple sclerosis has been initiated (ClinicalTrials.gov identifier: NCT02040298). The screening tool identified by Mei et al. could identify additional compounds that promote the remyelination of axons, thereby opening the door to the development of therapeutic options for patients with progressive multiple sclerosis.

Megan Cully

ORIGINAL RESEARCH PAPER Mei, F. et al. Micropillar arrays as a high-throughput screening platform for therapeutics in multiple sclerosis. Nature Med. 20, 954-960 (2014) 